\title{
Search for mixed-symmetry states of nuclei in the vicinity of the double-magic nucleus ${ }^{208} \mathrm{~Pb}$
}

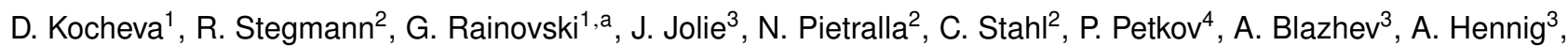 \\ C. Bauer ${ }^{2}$, Th. Braunroth ${ }^{3}$, M.P. Carpenter ${ }^{5}$, L. Cortes $^{2}$, A. Dewald ${ }^{3}$, M. Djongolov ${ }^{1}$, C. Fransen ${ }^{3}$, K. Gladnishki ${ }^{1}$, \\ R.V. F. Janssens ${ }^{5}$, V. Karayonchev ${ }^{3}$, M. Lettmann², C.J. Lister ${ }^{5}$, J. Litzinger ${ }^{3}$, Th. Möller ${ }^{2}$, C. Müller-Gatermann ${ }^{3}$, M. \\ Scheck $^{2, b}$, Ph. Scholz ${ }^{3}$, C. Schramm², P. Thöle ${ }^{3}$, V. Werner ${ }^{2}$, D. Wölk ${ }^{3}$, S. Zhu ${ }^{5}$, and P. Van Isacker ${ }^{6}$ \\ ${ }^{1}$ Faculty of Physics, St. Kliment Ohridski University of Sofia, 1164 Sofia, Bulgaria \\ ${ }^{2}$ Institut für Kernphysik, Technische Universität Darmstadt, D-64289 Darmstadt, Germany \\ ${ }^{3}$ Institut für Kernphysik, Universität zu Köln, D-50937 Köln, Germany \\ ${ }^{4}$ Bulgarian Academy of Sciences, Institute for Nuclear Research and Nuclear Energy, 1784 Sofia, Bulgaria \\ ${ }^{5}$ Physics Division, Argonne National Laboratory, 9700 South Cass Avenue, Argonne, Illinois 60439, USA \\ ${ }^{6}$ Grand Accélérateur National d'lons Lourds, CEA/DSM-CNRS/IN2P3, BP 55027, F-14076 Caen Cedex 5, France
}

\begin{abstract}
In this work we present the results from two experiments dedicated to search for quadrupolecollective isovector valence-shell excitation, the states with so-called mixed proton-neutron symmetry (MSS), in nuclei around the doubly magic nucleus ${ }^{208} \mathrm{~Pb} .{ }^{212} \mathrm{Po}$ was studied in an $\alpha$-transfer reaction. ${ }^{204} \mathrm{Hg}$ was studied in an inverse kinematics Coulomb excitation reaction on a carbon target. Both experiments provide indications for existence of one-phonon MSSs. Those are the first experimentally identified MSSs in the mass $A \approx 208$ region.
\end{abstract}

\section{Introduction}

States with mixed proton-neutron symmetry (MSSs) are the lowest-lying isovector excitations in the valence shell [1] for vibrational nuclei. Even though these states are defined in the framework of an algebraic model (IBM2) $[2,3]$, their properties simultaneously depend on the nuclear quadrupole collectivity, the underlying shell structure, and the proton-neutron balance of the wave function. Mixed-symmetry states are identified experimentally [4] by their strong isovector $M 1$ decay to the low-lying, fullysymmetric states (FSSs). The best examples of MSSs of stable nuclei are found in the mass $A \approx 90$ region $[4,5]$. In the last decade a large number of MSSs has been identified in the mass $\mathrm{A} \approx 130$ region [6-11]. Although experimental data for MSSs is relatively abundant, especially for properties of one-phonon $2_{1 ; \mathrm{ms}}^{+}$states, there are only few limited attempts to understand the mechanism that governs the formation and the evolution of these states with the number of nucleons. Apparently such mechanism should include the interplay between the single-particle structure, the quadrupole collectivity, and the isospin degrees of freedom. Heyde and Sau [12] have suggested a schematic model for formation of isovector excitations in the valence shell which, however, accounts only for the

\footnotetext{
ae-mail: rig@phys.uni-sofia.bg

${ }^{b}$ Present address: University of the West of Scotland, PA1 2BE Paisley, United Kingdom and Scottish Univ. Phys. Alliance, Glasgow G12 8QQ, Scotland
}

single-particle degree of freedom. Very recently, new experimental evidence for the formation mechanism of symmetric and mixed-symmetry low-lying quadrupole collective structures was reported for the $A \approx 90$ region [13]. It has been shown that the one-phonon FS and MS states result from coupling of the lowest 2-quasiparticle proton and neutron excitations to the Giant Quadrupole Resonance (GQR). Consequently, the $E 2$ strengths of these states are dominated by the GQR contributions while $M 1$ properties are determined by the leading valence shell configurations. Within this concept, the evolution of the $M 1$ strengths between MS and FSSs observed in $N=80$ isotones [7] and in the chain of xenon isotopes [8] can be understood; due to normalization of the wave functions, with increasing collectivity the GQR contribution will also increase at the expense of a reduction of the valence shell contribution which leads to a decrease of $M 1$ strength. This concept also demands that the strongest $M 1$ transitions between one-phonon MS and FSs in a given mass region occur in less collective vibrational nuclei. These nuclei are only 2 proton particles (holes) and 2 neutron particles (holes) away from double magic nuclei. Apparently, they will also have the simplest possible valence shell configurations. However, it also has been shown that the absolute $B\left(M 1 ; 2_{1 ; \mathrm{ms}}^{+} \rightarrow 2_{1}^{+}\right)$strength is highly sensitive to the proton-neutron balance of the wave functions; a mechanism dubbed Configurational Isospin Polarization (CIP) [14] occurs when proton and neutron amplitudes in the wave functions of one-phonon $2^{+}$states are not bal- 
anced, and either one dominates. The case of significant CIP is manifested by small absolute $M 1$ rates. The opposite case of vanishing CIP leads to a very strong $M 1$ transition between the one-phonon MS and FSSs. In this way the two lowest one-phonon, predominantly FS and MS, $2^{+}$states provide a tool to quantitatively study the $p-n$ balance of collective nuclear states.

The combined effect of low collectivity and vanishing CIP has been observed in ${ }^{132} \mathrm{Te}$ [10] - a nucleus which is two protons and two neutron holes away from the doubly magic nucleus ${ }^{132} \mathrm{Sn}$. It has been shown that the onephonon MSS of this nucleus, namely the $2_{2}^{+}$state, decays with an exceptionally strong $M 1$ transition to the FS $2_{1}^{+}$state [10]. The opposite case of well pronounced CIP is expected for ${ }^{136} \mathrm{Te}$ [15] due to the expectation that the $p-n$ exchange symmetry is strongly broken as Te and $\mathrm{Xe}$ isotopes depart from doubly magic ${ }^{132} \mathrm{Sn}$ [16]. Obviously, to better understand the interplay between collectivity and the isospin degrees of freedom in forming the low-lying isovector excitations, more cases of one-phonon MSSs in the vicinity of double-magic nuclei have to be identified and quantitatively studied. However, nuclei in the vicinities of doubly magic shell closures in which the onephonon $2_{1 ; \mathrm{ms}}^{+}$can be studied experimentally by conventional methods are scarce. In the mass $A \approx 130$ region, i.e. the mass region around the doubly magic nucleus ${ }^{132} \mathrm{Sn}$, all such nuclei are radioactive, including ${ }^{132} \mathrm{Te}$. A bit different is the situation around the doubly magic nucleus ${ }^{208} \mathrm{~Pb}$ where the nuclei having valence structures build on 2 proton particles (holes) - 2 neutron particles (holes) are accessible experimentally by conventional methods. ${ }^{204} \mathrm{Hg}$ is 2 protons holes and 2 neutron holes away from the double magic nucleus ${ }^{208} \mathrm{~Pb}$ and is stable. It can be studied in highly-inverse Coulomb excitation reactions [6]. ${ }^{212} \mathrm{Po}$, which has 2 protons and 2 neutrons more than ${ }^{208} \mathrm{~Pb}$, is accessible in $\alpha$-transfer reactions [17]. In this contribution we present the preliminary results from two such experiments that indicate existence of one-phonon MSSs in ${ }^{212} \mathrm{Po}$ and ${ }^{204} \mathrm{Hg}$.

\section{Identification of the one-phonon $2^{+}$mixed symmetric state of ${ }^{212} \mathrm{Po}$}

The experiment was performed at the FN Tandem facility of the University of Cologne. Excited states of ${ }^{212}$ Po were populated using the transfer reaction ${ }^{208} \mathrm{~Pb}\left({ }^{12} \mathrm{C},{ }^{8} \mathrm{Be}\right)$ at a beam energy of $62 \mathrm{MeV}$. The beam energy was chosen to be about $2 \mathrm{MeV}$ below the Coulomb barrier. The target was a self-supporting $10 \mathrm{mg} / \mathrm{cm}^{2}$ thick $\mathrm{Pb}$ foil enriched up to $99.14 \%$ in the isotope ${ }^{208} \mathrm{~Pb}$. The reaction took place in the reaction chamber of the Cologne coincidence plunger device [18] in which an array of solar cells was mounted at backward angles with respect to the beam direction in order to detect the recoiling back light reaction fragments. The solar cell array consisted of six $10 \mathrm{~mm} \times 10 \mathrm{~mm}$ cells placed at distance of about $15 \mathrm{~mm}$ between their centers and the target. The array covered an annular space between $116.8^{\circ}$ and $167.2^{\circ}$. The $\gamma$-rays from the decay of the excited states of ${ }^{212}$ Po were registered by 12 HPGe de- tectors mounted outside the plunger chamber in three rings at, on average, $12 \mathrm{~cm}$ from the target. Five detectors were positioned at $142.3^{\circ}$ with respect to the beam direction, another six formed a ring at $35^{\circ}$ and a single detector was placed at $0^{\circ}$. Data was taken in coincidence mode of at least one solar cell and one HPGe detector (particle- $\gamma$ ) or when at least two HPGe detectors $(\gamma-\gamma)$ were in coincidence.

The particle- $\gamma$ coincidence data was sorted in three matrices depending on the position of the HPGe detectors. A projection of the particle- $\gamma\left(@ 142^{\circ}\right)$ matrix is shown in Fig. 1(a). The $\gamma$-rays in coincidence with ${ }^{8} \mathrm{Be}$ (or $2 \alpha$ 's) are shown in Fig. 1(b). This spectrum is dominated by the $727 \mathrm{keV}$, the $405 \mathrm{keV}$ and the $223 \mathrm{keV}$ lines which are the $\gamma$ transitions from the first three yrast states of ${ }^{212}$ Po [17]. Most of the other $\gamma$ lines in the spectrum in Fig. 1(b) also originated from the decay of excited states of ${ }^{212}$ Po. A partial level scheme of the states of ${ }^{212}$ Po populated in the present experiment was constructed on the basis of the $\gamma-\gamma$ coincidence relationships. The level scheme is presented in Fig. 2 and is in agreement with the previously reported level schemes of ${ }^{212}$ Po [17, 19-22]. For the spinparities of the observed states we have adopted the values reported in previous studies [17, 19-22].

The levels presented in Fig. 2 are a part of those populated in another $\alpha$-transfer reaction, namely ${ }^{208} \mathrm{~Pb}\left({ }^{18} \mathrm{O},{ }^{12} \mathrm{C}\right)[17]$. In addition, we have populated two off-yrast $2^{+}$states at excitation energies of $1512 \mathrm{keV}$ and of $1679 \mathrm{keV}$, respectively [19]. These two states decay predominantly to the $2_{1}^{+}$state via the $785 \mathrm{keV}$ and the $952 \mathrm{keV}$ transitions, respectively [23]. These two transitions have well pronounced $M 1$ character with multipole mixing ratios of $+0.09(3)$ and $+0.65(50)$ [19], respectively. That makes these two $2^{+}$states potential candidates for the onephonon MSS of ${ }^{212} \mathrm{Po}$. The only missing piece of experimental information that is needed to verify this hypothesis are the short lifetimes of these levels.

Both $785 \mathrm{keV}$ and $952 \mathrm{keV}$ lines indeed show well pronounced Doppler shapes which allows for lifetimes determination of the $2_{2}^{+}$and $2_{3}^{+}$states in ${ }^{212}$ Po by means of the Doppler Shift Attenuation Method (DSAM) (c.f. Ref. [24] and references therein). We have performed two parallel analyses of the line shapes; in the first analysis, labelled here Analysis I, we used a Monte Carlo (MC) simulation by means of a modified [25, 26] version of the program DESASTOP [27] in order to describe the slowing down of the recoiling nuclei. The electronic stopping powers used were obtained from the Northcliffe and Schilling tables [28] with corrections for the atomic structure of the medium, as discussed in Ref. [29]. An empirical reduction of $f_{n}=0.7$ was applied [30] to downscale the nuclear stopping power predicted by the theory of Lindhard, Scharff, and Schiøtt [31]. The second analysis, labelled here Analysis II, uses an integrated software named APCAD (Analysis Program for Continuous Angle DSAM) [32]. In APCAD, the slowing down process is simulated by GEANT4 [33] with the electronic stopping process modelled in the same way as in Analysis I. On the other side, APCAD adopts a simpler approach to mod- 

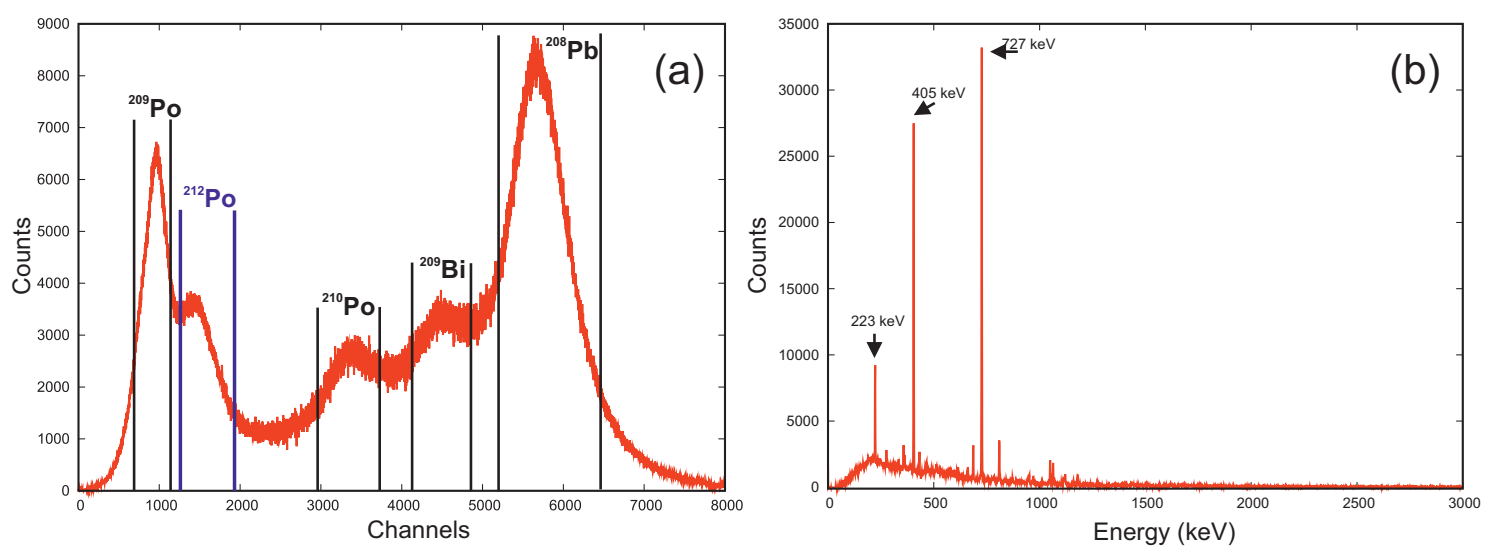

Figure 1. (Color online) (a) The projection of the particle- $\gamma\left(@ 142^{\circ}\right)$ matrix. The vertical lines represent groups of particles found to be in coincidences with the $\gamma$ rays from the indicated nuclei. (b) The $\gamma$-ray spectrum in coincidences with the group of particles indicated as "212 $\mathrm{Po"} \mathrm{in} \mathrm{panel} \mathrm{(a).}$

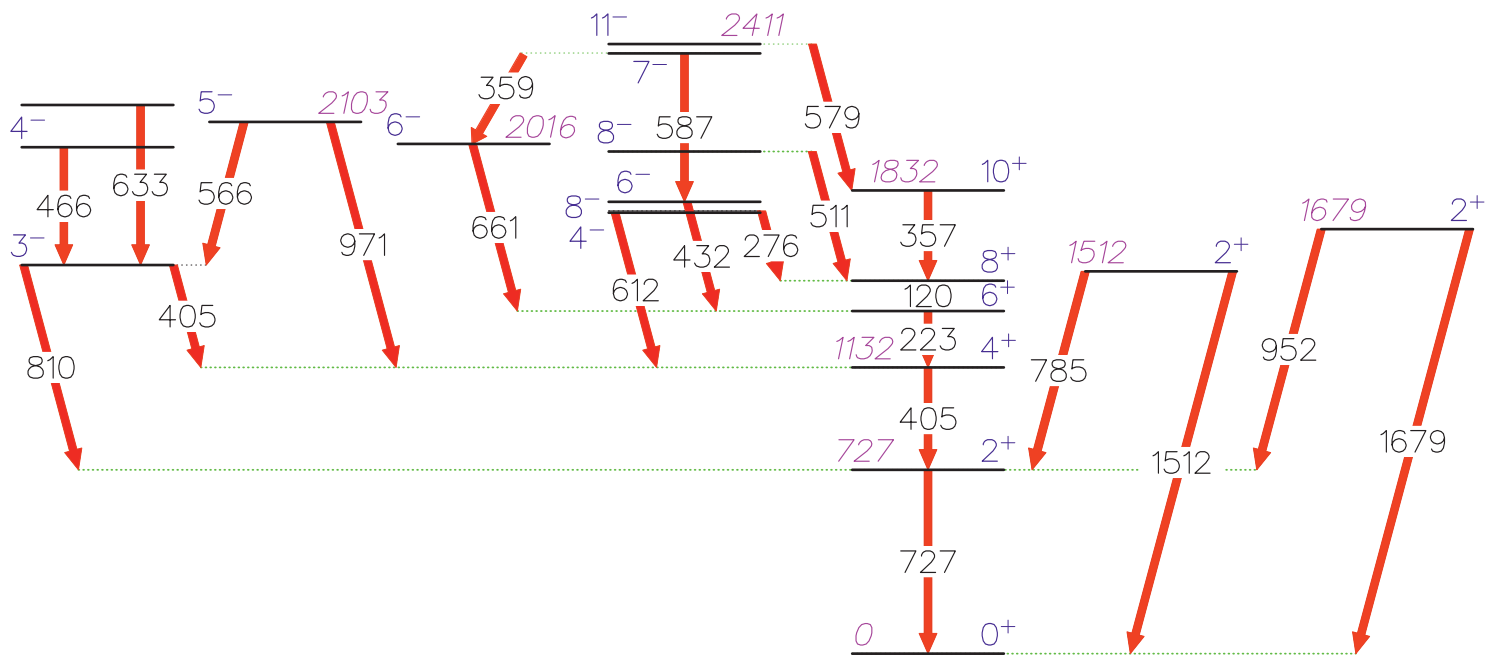

Figure 2. (Color online) Partial level scheme of ${ }^{212}$ Po obtained in the present work.

elling the nuclear stopping process, compared with the completely discrete approach used in Analysis I; in Analysis II the angular straggling due to nuclear collisions is modelled discretely by means of MC simulation while the corresponding energy loss is considered to emerge as a result from a continuous process and the nuclear stopping powers were taken from SRIM2013 [34] and reduced by $40 \%$. Both analyses account for the response of the HPGe detectors, for the experimental geometry, and for the restrictions on the reaction kinematics imposed by the solar cell array. The feeding histories of the levels of interest were determined from the $\gamma-\gamma$ coincidence data. Slow feeding was introduced and fitted in the analyses only if the analysed transitions were observed in coincidence with transitions from higher-lying states. In the opposite case, only very fast feeding which can be associated with direct population of the levels of interest, was considered.

Under the above assumptions both analyses produced similar results. For example, the lifetime of the $6^{-}$state at $2016 \mathrm{keV}$ (see Fig. 2) is known to be $0.49(16)$ ps [17]. The lifetime of this level derived in our analyses from the line shape of the $661.3 \mathrm{keV}$ transition, with a feeding history similar to the one used in Ref. [17] (58\% fast feeding and $42 \%$ slow feeding), is $0.50(4)$ ps in Analysis I and $0.47(3) \mathrm{ps}$ in Analysis II. The lifetimes of the $2_{2}^{+}$state at $1512 \mathrm{keV}$ and $2_{3}^{+}$state at $1679 \mathrm{keV}$ were extracted from the line shapes of the $785 \mathrm{keV}$ and the $952 \mathrm{keV}$ transitions (see Fig. 2), respectively. Both of these transitions are in coincidence with $727 \mathrm{keV}$ transition, only. In Fig. 3, an example of the line-shape fit for the $952 \mathrm{keV}$ transition is presented. The final lifetimes together with the available spectroscopic information and the resulting transition strengths are summarized in Table 1.

As seen from Table 1 the $2_{2}^{+}$state of ${ }^{212}$ Po at $1512 \mathrm{keV}$ excitation energy decays with a sizeable $M 1$ transition to the $2_{1}^{+}$state. This allows us to conclude that the $2_{2}^{+}$state of ${ }^{212} \mathrm{Po}$ is, at least, a fragment of the one-phonon MSS. To our knowledge this is first MSS in mass $A=208$ region firmly identified on the basis of measured strong $M 1$ decay. 
Table 1. Properties of the $2_{2}^{+}$and the $2_{3}^{+}$states of ${ }^{212} \mathrm{Po}$.

\begin{tabular}{|c|c|c|c|c|c|c|c|c|}
\hline$E_{\text {level }}(\mathrm{keV})$ & $J^{\pi}$ & $\tau(\mathrm{ps})^{\mathrm{a}}$ & $E_{\gamma}(\mathrm{keV})$ & $I_{\gamma}{ }^{\mathrm{b}}$ & $\alpha^{\mathrm{c}}$ & $J_{\text {final }}^{\pi}$ & $\delta^{\mathrm{d}}$ & Transition strength $^{\mathrm{e}}$ \\
\hline \multirow[t]{3}{*}{1512} & $2_{2}^{+}$ & $0.67(5)$ & 1512.7 & $26(3)$ & & $0_{1}^{+}$ & & $B(E 2)=32(4)$ \\
\hline & & & 785.4 & $100(1)$ & $0.0408(2)$ & $2_{1}^{+}$ & $0.09(3)$ & $B(M 1)=0.133(9)$ \\
\hline & & & & & & & & $B(E 2)=25(16)$ \\
\hline \multirow[t]{3}{*}{1679} & $2_{3}^{+}$ & $0.68(2)$ & 1679.7 & $35(8)$ & & $\overline{0_{1}^{+}}$ & & $B(E 2)=23(5)$ \\
\hline & & & 952.1 & $100(19)$ & $0.020(5)$ & $2_{1}^{+}$ & $0.65(50)$ & $B(M 1)=0.049(17)$ \\
\hline & & & & & & & & $B(E 2)=331(306)$ \\
\hline
\end{tabular}

${ }^{a}$ Preliminary results from the present experiment.

${ }^{\mathrm{b}}$ From Ref. [23].

${ }^{\mathrm{c}}$ Total electron conversion coefficients. From Ref. [23].

${ }^{\mathrm{d}}$ From Ref. [20].

${ }^{\mathrm{e}} B(E 2)$ values are given in $\mathrm{e}^{2} \mathrm{fm}^{4}\left(1 \mathrm{~W} . \mathrm{u} .=75.09 \mathrm{e}^{2} \mathrm{fm}^{4}\right)$, and the $B(M 1)$ values are given in $\mu_{\mathrm{N}}^{2}$. In the calculations for the transitions strengths we have assumed vanishing $\alpha$-decay branches.

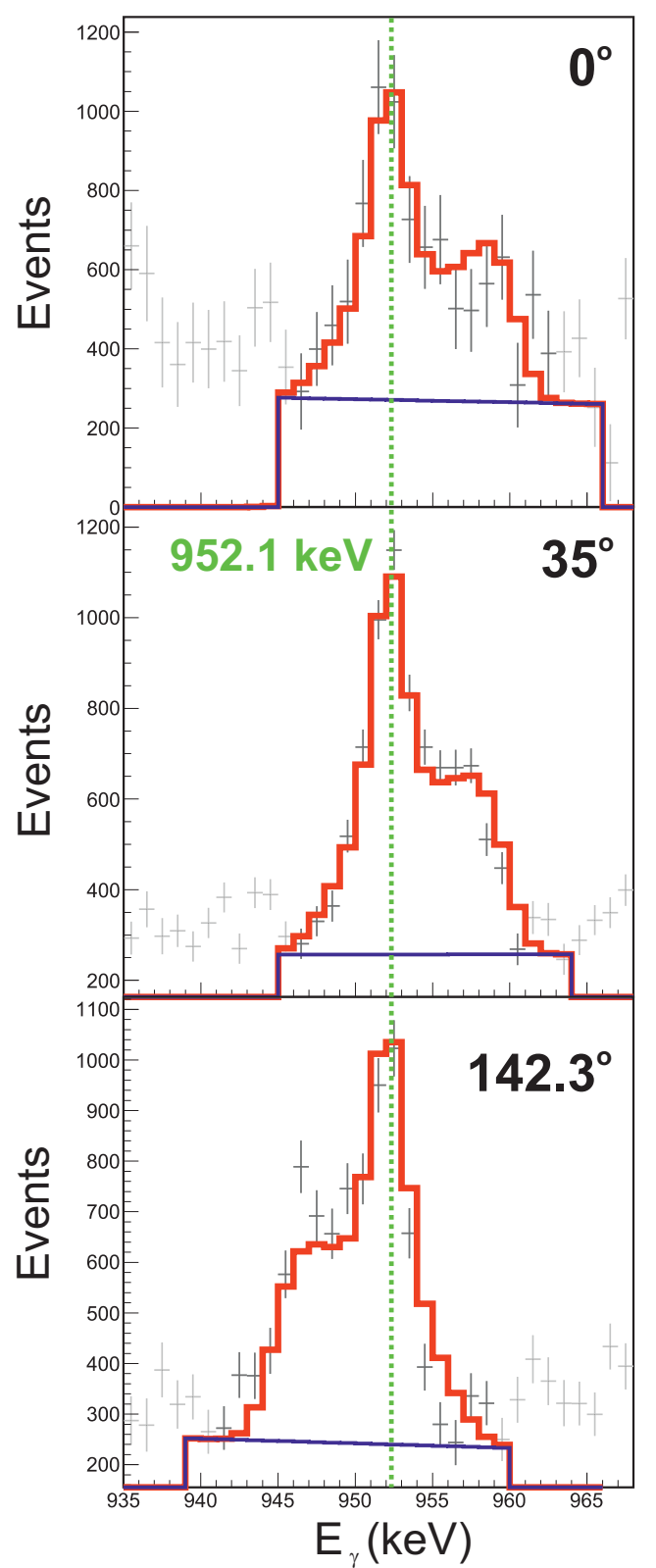

Figure 3. (Color online) An example of line-shape fit of the $952.1 \mathrm{keV}$ transitions obtained with the program APCAD. The vertical dotted line shows the position of the unshifted peak.

\section{Search for the one-phonon $2^{+}$mixed-symmetric state of ${ }^{204} \mathrm{Hg}$}

Low-lying states of ${ }^{204} \mathrm{Hg}$ were populated and studied in a Coulomb excitation reaction. The experiment was carried out at Argonne National Laboratory in inverse kinematics. The pulsed $(12 \mathrm{MHz}){ }^{204} \mathrm{Hg}$ beam, with intensity of $\approx 1.3 \mathrm{pnA}\left(\sim 8 \times 10^{9}\right.$ ions/s $)$, was delivered by the ATLAS accelerator. It was incident on a $1 \mathrm{mg} / \mathrm{cm}^{2}$ thick ${ }^{12} \mathrm{C}$ target with an energy of $890 \mathrm{MeV}$. This energy corresponds to $\approx 82 \%$ of the Coulomb barrier for the reactions of ${ }^{204} \mathrm{Hg}$ ions on a ${ }^{12} \mathrm{C}$ target [35]. The deexcitation $\gamma$ rays, following Coulomb excitation of the projectile, were detected with the Gammasphere array [36] which consisted of 100 HPGe detectors. Gammasphere was used in singles mode, resulting in an average counting rate of 6800 counts-per-second (cps), while the room background was about $160 \mathrm{cps}$. A total of $4.4 \times 10^{8}$ events of $\gamma$-ray fold 1 or higher was collected in about 18 hours. The contribution of the room background was eliminated in the off-line sort by correlating the $\gamma$ rays with the accelerator radio-frequency (RF) signal. The final spectrum, which is a difference between the "beam-on" (with respect to the RF) spectrum and the "beam-off" spectrum, scaled to eliminate the $1461 \mathrm{keV}$ room background transition following the decay of ${ }^{40} \mathrm{~K}$, is shown in Fig. 4. All $\gamma$ rays in the spectrum originate from ${ }^{204} \mathrm{Hg}$ nuclei recoiling with $v / c \approx 8.4 \%$. Most of these $\gamma$ rays have already been identified in the decay scheme of ${ }^{204} \mathrm{Hg}[37,38]$. About $1.4 \%$ of the data have $\gamma$-ray fold higher than 1. These events were sorted into a $\gamma-\gamma$ coincidence matrix. This matrix was used to construct the level scheme of ${ }^{204} \mathrm{Hg}$ that is presented in Fig. 5.

The spectrum in Fig. 4 is dominated by the X-rays of mercury and the $437 \mathrm{keV}$ transition which decays the $2_{1}^{+}$state in ${ }^{204} \mathrm{Hg}$. There are several other intensive transitions and amongst them $1511 \mathrm{keV}$ is a notable one. This transition connects the $2_{4}^{+}$state at excitation energy of $1948 \mathrm{keV}$ to the $2_{1}^{+}$state. Preliminary results for the angular distribution of this transition indicated almost pure dipole character. This makes the $2_{4}^{+}$level at $1948 \mathrm{keV}$ a potential candidate for the one-phonon MSS. This is additionally supported by the fact that the $3_{1}^{-}$state decays quite 


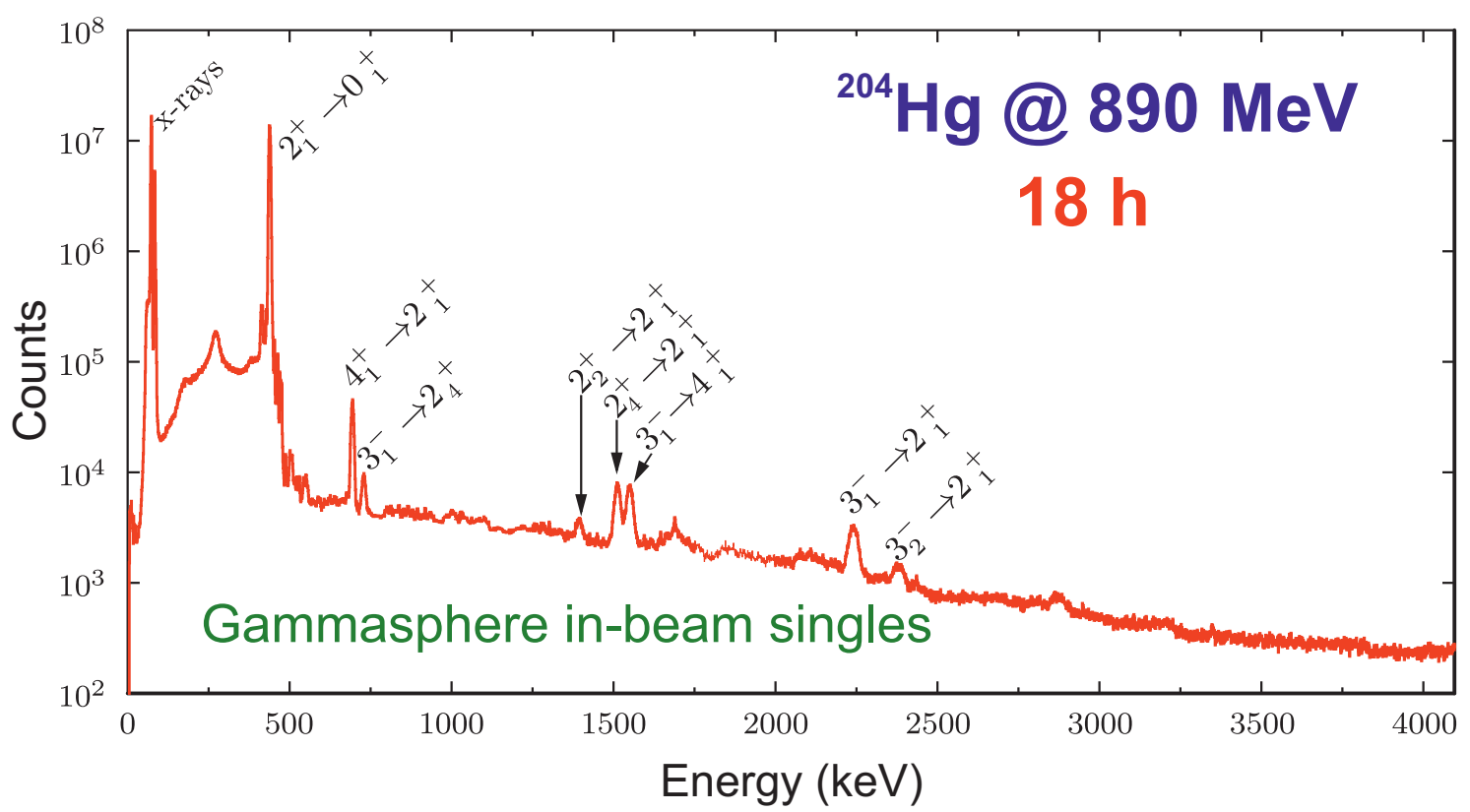

Figure 4. (Color online) Background-subtracted, Doppler-corrected $\gamma$-ray spectrum of ${ }^{204} \mathrm{Hg}$ observed with Gammasphere after Coulomb excitation on a carbon target. The weak transitions in ${ }^{204} \mathrm{Hg}$ are not indicated.

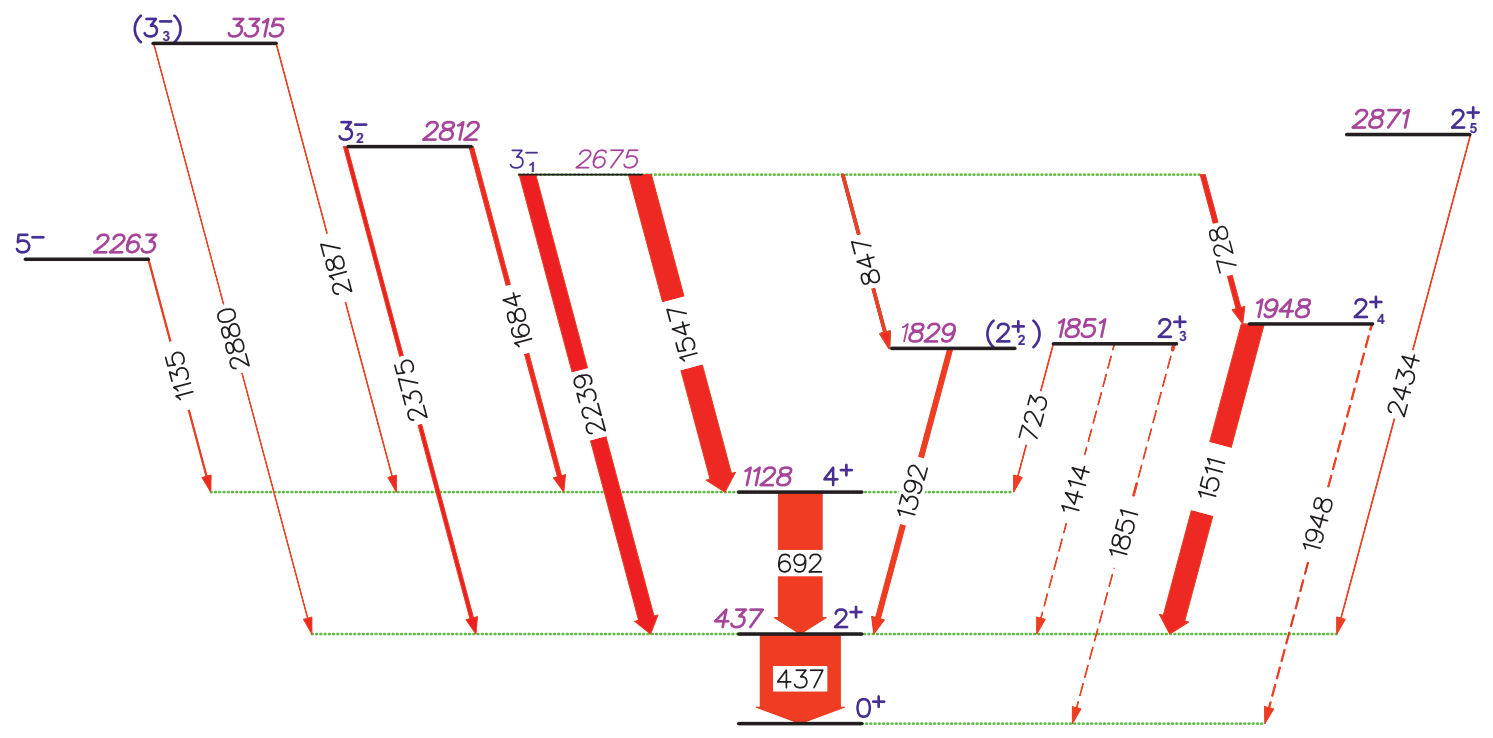

Figure 5. (Color online) Partial level scheme of ${ }^{204} \mathrm{Hg}$ obtained in the present work. The thickness of the arrows are proportional to the $\gamma$-ray intensities. The intensity of the $437 \mathrm{keV}$ transition is downscaled by a factor of 1000 for presentation purposes.

intensively to the $2_{4}^{+}$state via a $728 \mathrm{keV}$ transition (c.f. Fig. 4 and Fig. 5). The relative $\gamma$-ray yields with respect to the $2_{1}^{+}$state measure the relative Coulomb excitation (CE) cross-sections. Predictions of the Winther-De Boer theory [39] were fitted to the experimental yields with the multiple CE code GOSIA [40] by using the known value $B\left(E 2 ; 0_{1}^{+} \rightarrow 2_{1}^{+}\right)=0.427(7) \mathrm{e}^{2} \mathrm{~b}^{2}$ [41] for setting the absolute scale. The fitting procedure accounts for the energy loss of the beam in the target, and the size of the unknown quadrupole moments [6] and provides a set of matrix elements describing the experimentally observed relative CE cross-sections. The preliminary results indicate that the
$M 1$ strength of the decay of the $2_{4}^{+}$state to the $2_{1}^{+}$state is about $0.5 \mu_{N}^{2}$. This unexpectedly large $B(M 1)$ value allows us to consider the $2_{4}^{+}$state of ${ }^{204} \mathrm{Hg}$ as a single isolated one-phonon MSS.

\section{Conclusion}

In summary, we have presented two experiments dedicated to search of MSSs of nuclei in the vicinity of the doubly magic nucleus ${ }^{208} \mathrm{~Pb}$. The preliminary results from these investigations indicate the existence of one-phonon MSSs of ${ }^{212} \mathrm{Po}$ and ${ }^{204} \mathrm{Hg}$. These are the first experimentally 
identified MSSs in the mass $A \approx 208$ region and they call for theoretical explanations how the states of isovector nature are formed and evolve in this mass region.

\section{Acknowledgements}

We thank A. Astier for useful discussions regarding the utilization of the DSAM in transfer reactions. G.R. acknowledges the support from the Alexander von Humboldt foundation. This work was supported by the partnership agreement between the University of Cologne and University of Sofia, by the DAAD German-Bulgarian exchange program under grants No. PPP57082997 and No. DNTS/01/05/2014, by the DFG under grant Pi393/2-3, and by the BMBF under grants No. 05P12RDCI8 and No. 05P15RDCIA.

\section{References}

[1] N. Lo Iudice and F. Palumbo, Phys. Rev. Lett. 41, 1532 (1978)

[2] F. Iachello, Phys. Rev. Lett. 53, 1427 (1984)

[3] F. Iachello and A. Arima, The Interacting Boson Model (Cambridge University Press, Cambridge, 1987)

[4] N. Pietralla, P. von Brentano, A.F. Lisetskiy, Prog. Part. Nucl. Phys. 60, 225 (2008), and references therein.

[5] K. Heyde, P. von Neumann-Cosel, A. Richter, Rev. Mod. Phys. 82, 2365 (2010)

[6] G. Rainovski et al., Phys. Rev. Lett. 96, 122501 (2006)

[7] T. Ahn et al., Phys. Lett. B 679, 19 (2009)

[8] L. Coquard et al., Phys. Rev. C 82, 024317 (2010)

[9] K.A. Gladnishki et al., Phys. Rev. C 82, 037302 (2010)

[10] M. Danchev et al., Phys. Rev. C 84, 061306(R) (2011)

[11] T. Ahn et al., Phys. Rev. C 86, 014303 (2012)

[12] K. Heyde and J. Sau, Phys. Rev. C 33, 1050 (1986)

[13] C. Walz et al., Phys. Rev. Lett. 106, 062501 (2011)

[14] J.D. Holt, N. Pietralla, J.W. Holt, T.T.S. Kuo, G. Rainovski, Phys. Rev. C 76, 034325 (2007)

[15] D. Bianco, N. Lo Iudice, F. Andreozzi, A. Porrino, F. Knapp, Phys. Rev. C 88, 024303 (2013)

[16] D.C. Radford et al., Phys. Rev. Lett. 88, 222501 (2002)

[17] A. Astier, P. Petkov, M.-G. Porquet, D.S. Delion, P. Schuck, Phys. Rev. Lett. 104, 042701 (2010); A. Astier, P. Petkov, M.-G. Porquet, D.S. Delion, P. Schuck, Eur. Phys. J. A 46, 165 (2010)
[18] A. Dewald, O. Möller, P. Petkov, Prog. Part. Nucl. Phys. 67, 786 (2012)

[19] B. Bengtson et al., Nucl. Phys. A 378, 1 (1982)

[20] A.R. Poletti et al., Nucl. Phys. A 473, 595 (1987)

[21] Zs. Podolyák et al., Nucl. Instr. Meth. A 511, 354 (2003)

[22] A.B. Garnsworthy et al., J. Phys. G: Nucl. Part. Phys. 31, S1851 (2005)

[23] E. Browne, Nucl. Data Sheets 104, 427 (2005)

[24] T.K. Alexander and J.S. Forster, Adv. Nucl. Phys. 10, 197 (1978)

[25] P. Petkov et al., Nucl. Phys. A 640, 293 (1998)

[26] P. Petkov et al., Nucl. Instr. Meth. A 431, 208 (1999)

[27] G. Winter, ZfK Rossendorf Report ZfK-497, 1 (1983); G. Winter, Nucl. Instr. Meth. 214, 537 (1983)

[28] L.C. Northcliffe and R.F. Schilling, At. Data Nucl. Data Tables 7, 233 (1970)

[29] J.F. Ziegler and J.P. Biersack, in Treatise on HeavyIon Science: Vol. 6: Astrophysics, Chemistry, and Condensed Matter, ed. D.A. Bromley (Plenum Press, New York, 1985), 95.

[30] J. Keinonen, AIP Conf. Proc. 125, 557 (1985)

[31] J. Lindhard, M. Scharff, H.E. Schiøtt, Mat. Fys. Medd. Dan. Vid. Selsk. 33, No. 14 (1963)

[32] C. Stahl, New methods for the $\gamma$-ray spectroscopy with position-sensitive detector systems, $\mathrm{PhD}$ thesis, Technische Universität Darmstadt (2015)

[33] S. Agostinelli et al., Nucl. Instr. Meth. A 506, 250 (2003)

[34] J.F. Ziegler, M.D. Ziegler, J.P. Biersack, Nucl. Instr. Meth. B 268, 1818 (2010)

[35] D. Pelte and D. Schwalm, in Heavy Ion Collisions, Vol. 3, ed. R. Bock (North-Holland, Amsterdam, 1982), p. 1.

[36] I.Y. Lee, Nucl. Phys. A 520, 641c (1990)

[37] R.A. Gatenby, E.W. Kleppinger, S.W. Yates, Nucl. Phys. A 492, 45 (1989)

[38] A. Hogenbirk, H.P. Blok, M.N. Harakeh, Nucl. Phys. A 524, 251 (1991)

[39] A. Winther and J. De Boer, in Coulomb Excitation, eds. K. Alder, A. Winther (Academic Press, New York, 1966), p. 303.

[40] T. Czosnyka, D. Cline, C. Wu, Bull. Am. Phys. Soc. 28, 745 (1983)

[41] S. Raman et al., At. Data Nucl. Data Tables 78, 1 (2001) 